\title{
Evaluation of depression in patients with bone
}

\section{metastasis before and after radiotherapy}

\author{
Feryal Karaca ${ }^{{ }^{\star}}$, Selahattin Menteş ${ }^{1}$, Çiğdem Usul Afşar ${ }^{2}$, Yaşar Sertdemir ${ }^{3}$, Timuçin Çil ${ }^{4}$ \\ 1 Adana Sehir Hastanesi Sağlık Bilimleri University Radiation Oncology \\ 2Istanbul Bakırköy Acıbadem Hospital Medical Oncology \\ ${ }^{3}$ Cukurova University Faculty of Medicine Department of Biostatistics \\ ${ }_{4}$ Adana Şehir Hastanesi Sağlık Bilimleri University Medical Oncology
}

\begin{abstract}
Bone is the third most common site of metastasis after lung and liver. Most of the bone metastasis come from either prostate or breast cancers. The incidence of developing bone metastasis in advanced diseases is quite high. Besides; depression is common in cancer patients with metastasis. Beck Depression Inventory is used in assessment of depression in patients. External beam radiation therapy is an effective technique in achieving pain control in patients with bone metastasis.

One hundred patients were included in the study according to their primary diseases. Patients were divided into four groups and Beck depression test was performed twice to determine the depression levels of the patients before and after radiotherapy. Before radiotherapy patients were given Beck-0 and after radiotherapy they were given Beck-1

Results of Beck -0 and Beck -1 , were statistically significant among any group itself and among all groups $\mathrm{p}<0.001$. Those scores were also significant for number of bone metastasis and number of radiotherapy treatment $\mathrm{p}<0.001$.

Depression is a common disease among the oncologhy patients, and can affect them, negatively. Beck scale scores after radiotheraphy have been found to be higher than those before radiotheraphy.
\end{abstract}

Key Words: Bone, neoplasm metastasis, radiotherapy, depression

\section{Introduction}

Cancer is the second leading cause for death after ischemic heart disease in developed countries. Metastasis occurs in advanced cancer patients due to loss of intercellular cohesion, cell migration and reemergence of angiogenesis. Circulation is needed for metastasis (1). In patients with primary breast cancer and prostate cancer, bone is the third commonly metastased site after lung and liver (2). Bone metastasis occur in $65-75 \%$ of advanced breast cancer, $65-75 \%$ of prostate cancer, $60 \%$ of thyroid cancer, $30-40 \%$ of lung cancer, $40 \%$ of bladder cancer, $20-25 \%$ of renal cell carcinoma and $14-45 \%$ of melanoma (3). The presence of bone metastasis in any given patient is indicative of widespread disease and shortness of the survival. A multidisciplinary approach is required after bone metastasis has occurred in the patient. The patient with bone metastasis develops morbidity-enhancing symptoms such as severe pain, limitation of motion, bone fracture, spinal cord compression and pansitopenia (4). Radiotherapy (RT) is preferred to localized bone metastasis as well as chemotherapy, hormonal therapy, and surgery. RT in localized bone metastasis provides $70 \%$ more pain control compared to standardized protocols. Different doses and fractionations are given for palliation of patients with bone metastasis and bone pain, but the need for retreatment was remerkably higher in patients treated with a single fraction regimen. Treating Patients with bone metastasis at a dose of $30 \mathrm{~Gy}$ in 10 fractions is awidespread and standard (5).

Depression is common in cancer patients $(6,7,8)$. Presence of depression in cancer patients and reduces the quality of life (9). Its diagnosis and treatment in cancer patients are difficult (10). The Beck Depression Inventory (BDI) is the most commonly applied scale of depression worldwide. It is an actual depression test used to evaluate depression in cancer patients with different cancer types $(11,12,13)$. Its validity and sensitivity are quite high. BDI includes 21 questions. Scores from 0 to 63 are given. Test scores less than 10 indicate none depression, and scores between 10-18 indicate minimal depression, between 10-18 indicate moderate depression, between 19-29 indicate moderate to severe depression, and between 30-63 indicate severe depression. Turkish validity has been made by Hisli (14). 
Karaca et al / Depression with bone metastasis

Table 1. Ages and primary diseases of patients and scores of Beck - 0 and Beck- 1

\begin{tabular}{|c|c|c|c|c|c|}
\hline \multicolumn{6}{|c|}{ Primer } \\
\hline & $\begin{array}{l}\text { Lung } \\
\mathrm{n}=25\end{array}$ & $\begin{array}{l}\text { Breast } \\
n=35\end{array}$ & $\begin{array}{c}\text { Prostate } \\
n=22\end{array}$ & $\begin{array}{l}\text { Other } \\
\mathrm{n}=18\end{array}$ & \multirow{3}{*}{$\mathrm{p}$} \\
\hline & Mean \pm sd & Mean \pm sd & Mean \pm sd & Mean \pm sd & \\
\hline & Median(Min,Max) & $\begin{array}{l}\text { Median ( } \\
\text { Min,Max) }\end{array}$ & $\begin{array}{l}\text { Median ( } \\
\text { Min,Max) }\end{array}$ & Median(Min,Max) & \\
\hline \multirow{2}{*}{ Age } & $64.56 \pm 6.13$ & $65.69 \pm 5.85$ & $67.0 \pm 6.99$ & $67.06 \pm 6.32$ & \multirow[t]{2}{*}{0.495} \\
\hline & $65(55,75)$ & $66(55,76)$ & $67.50(58,78)$ & $69(56,77)$ & \\
\hline \multirow{2}{*}{ Beck Before RT } & $53.56 \pm 11.13$ & $31.20 \pm 11.13$ & $37.14 \pm 15.35$ & $42.89 \pm 14.56$ & \multirow[t]{2}{*}{$<0.001$} \\
\hline & $55(19,61)$ & $28(15,58)$ & $35(14,58)$ & $52(19,58)$ & \\
\hline \multirow{2}{*}{ Beck After RT } & $59.58 \pm 8.54$ & $36.71 \pm 13.82$ & $42.86 \pm 18.25$ & $49.28 \pm 15.62$ & \multirow[t]{3}{*}{$<0.001$} \\
\hline & $61(22,63)$ & $35(13,63)$ & $46(13,63)$ & $60.5(23,63)$ & \\
\hline pBeck & $<0.001$ & $<0.001$ & $<0.001$ & $<0.001$ & \\
\hline
\end{tabular}

For all groups no association was found between Beck scores and age $(\mathrm{p}=0.495)$. Beck-0 and Beck-1 scores were significant for all groups $\mathrm{p}<0.001$

Table 2. Changes in test scores with number of metastasis and number of RT received

\begin{tabular}{lcccc}
\hline & \multicolumn{3}{c}{ Number of bone metastasis and the number RTs } & \multirow{2}{*}{$\mathrm{p}$} \\
\cline { 2 - 4 } & 1 Mean \pm sd & 2 Mean \pm sd & $3+$ Mean \pm sd & $<0.001$ \\
\cline { 2 - 4 } Beck- 0 & $28.43 \pm 8.81$ & $55.16 \pm 2.09$ & $55.20 \pm 3.91$ & $<0.001$ \\
Beck-1 & $33.73 \pm 12.14$ & $61.74 \pm 0.87$ & $61.60 \pm 1.12$ & \\
pBeck & $<0.001$ & $<0.001$ & $<0.001$ & \\
\hline
\end{tabular}

When Beck-0 and Beck-1 values were separately evaluated according to bone metastasis and RT counts $(1,2,3+)$ depression score change was found statistically significant according to the change of metastasis number and RT number $\mathrm{p}<0.001$.The change in depression score was found to be statistically significant when Beck- 0 and Beck- 1 were compared before and after RT ( depending on the number of bone metastasis and the number RTs) was found to be statistically significant $\mathrm{p}<0.001$. As number of metastasis and number of RT increased the Beck- 0 and Beck -1 scores also increased. $\mathrm{p}<0.001$

\section{Material and Method}

One hundred patients (46 female, 54 male) with bone metastasis of different cancer origins were enrolled in the study and given Beck- 0 before radiotherapy and Beck-1 after radiotherapy between April 2016 and September. 2017. Beck depression test is only given to patients who have been under RT for 10 days.

100 patients between 55-77 years of age (55-76 female, 55-77 male) who completed both tests were recruited. Patients with bone metastasis were divided into four groups as lung cancer, breast cancer, prostate cancer and 'other cancers' group according to their primary disease. Lung cancer group had 25 patients, breast cancer group had 35 patients, prostate cancer group had 22 patients and 'other cancers' had 18 patients (gastric cancer 8 patients, ovarian cancer 3 patients, colon cancer 1 patient, pancreatic cancer 1 patient, sarcoma 2 patients and endometrial cancer 3 patients).

Finally changes in Beck- 0 and Beck-1 scores were evaluated according to the number of metastasis and number of radiotherapy received (Table 2).
Radiotherapy: All patients were given RT as palliative doses and conformol RT technique was used 10X300 cGy total 3000cGy.

Psychological support: During the RT patients were followed and discussed and the patients who had Beck depression scores over 17 were consulted to psychiatry. Patients who were referred to psychiatry were given antidepressants. Written consent of the patients were taken and the study was approved by Cukurova University Ethics Committee.

Statistical Analysis: All analyses were performed using IBM SPSS Statistics Version 20.0 statistical software package. Continuous variables were summarized as mean and standard deviation and as median and minimum-maximum where appropriate. The paired samples t-test was used to compare two related (paired) continuous variables and Oneway ANOVA was used for comparison of more than two groups. Kaplan-Meier and Cox regression analysis was performed to compare time from metastasis to death. The statistical level of significance for all tests was considered to be 0.05 . 
Table 3. Survi and death risk of metastatic lung cancer patients with Beck scores more than 6

\begin{tabular}{llll}
\hline & \multicolumn{2}{l}{ Lung cancer } & \\
\cline { 2 - 3 } & $\leq 5$ & $6+$ & Mean 95\%CI \\
\cline { 2 - 4 } Time metastasis to death & Mean 95\%CI & $321(304-338)$ & 0.025 \\
\hline
\end{tabular}

3.45(1.06-11.23) $\mathrm{p}=0.004$ cox regression increase of the risk log rank lifetime $\mathrm{p}=0.025$.

Subgroups analysis showed that patients with metastatic lung cancer display doubled death risk and the shortest survi, especially when Beck scores before RT and after RT exceed 6

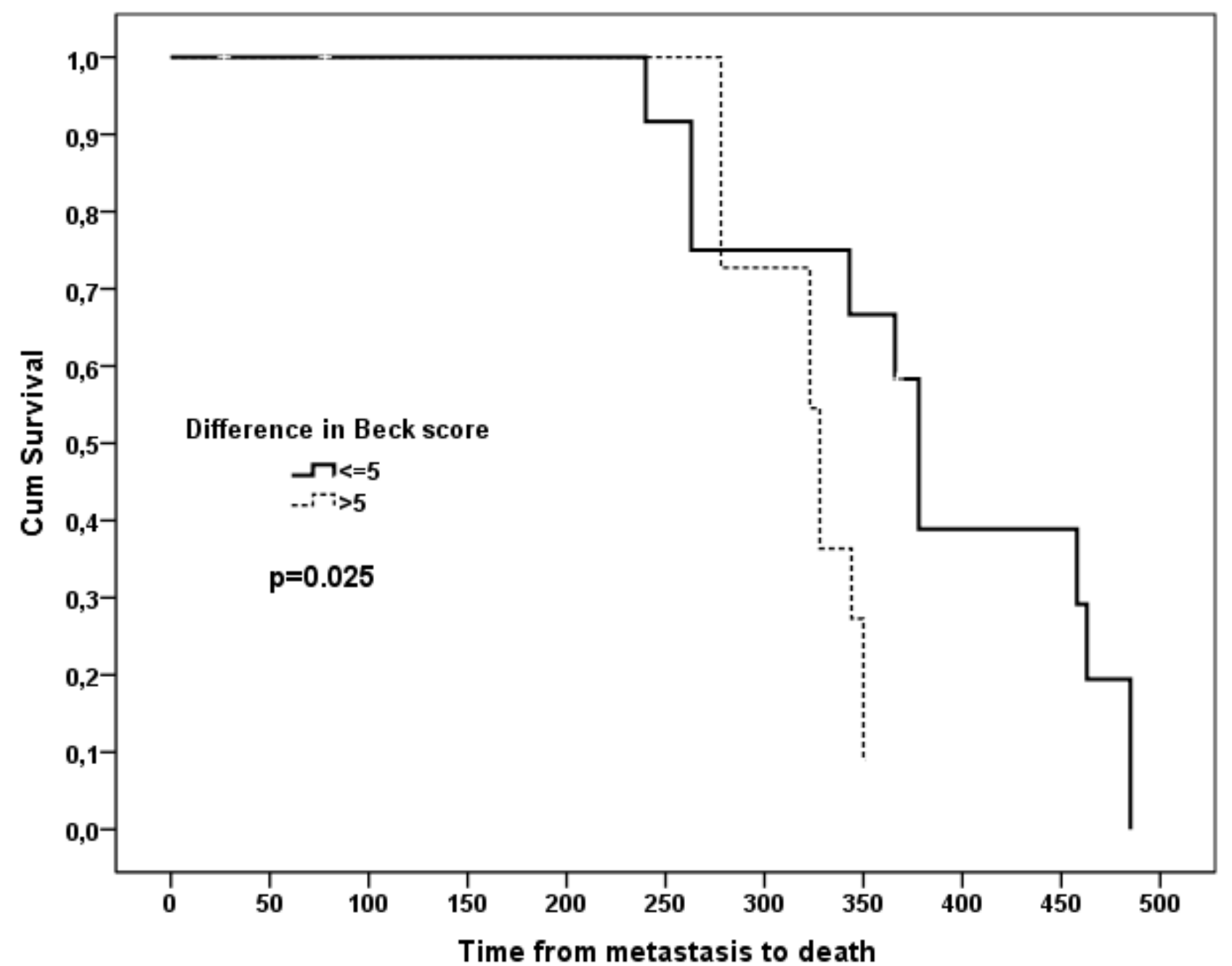

Fig. 1.Time from metastasis to death for lung cancer patients with Beck score difference $>6$

\section{Results}

Ages of patients with bone metastasis, primary diseases of the patients and results of Beck- 0 and Beck -1 before and after RT is given in Table 1 .

\section{Discussion}

The bone metastasis of any given cancer shows that the disease is advanced and indicates poor prognosis. It can only be palliatively treated by external beam RT which is also the best option for pain management.
Palliative RT is a localized treatment and is the standard approach. The aim of RT is to relieve patients' pain as quick as possible (15). We evaluated prospective depression scales with $\mathrm{RT}$ in patients with different primary diseases. In our study we found that age was not associated with Beck scores of RT patients in lung, breast, prostate and a group of cancers (Table 1). It can be thought that level of depression caused by a cancer is independent of the age of the patients. And the level of depression seems to rise equally after RT. The patients with Beck-0 lung cancer bone metastasis have very high severe depression scores. The patients with breast 
cancer bone metastasis displayed Beck-0 scores between moderate and severe depression range. Patients with prostate cancer, and bone metastasis displayed Beck-0 scores, between moderate and severe. However Beck-0 depression scores for 'other cancers' group is found to be severe. We think it is normal to assume that lung cancer patients to have greater concerns for their disease than the other cancer groups and develop more depression (Table 1). Between groups difference between Beck- 0 and Beck-1 was found to be significant (Table1). All the patients in the groups displayed severe depression scores for Beck -1 (Table 1).

Beck- 0 and Beck -1 scores were significant for all groups (Table1). It is possible to assume that for all cancer groups advanced therapies increase patient depression. The patients' ages were between 55 and 78. If it is thought that elderly cancer patients tend to have widespread metastatic disease as opposed to younger patients it is logical to suppose them to have greater depressive scores which was not found in our study even though they respond to chemotherapy and RT poorly. There are numerous other possible parameters for development of depression in a group of cancer patients and it is impossible to imagine and screen for all of them. Ming-Szu Hung et all. have shown that depression was more prevelant in lung cancer patients compared to normal controls. They have also stated that depression occurred regarding to age, gender, chronic diseases and life conditions (16) Our study showed that Beck-0 score increased when number of RT increased for all cancer groups (Table 2). Likewise Beck -1 score also increased as RT number increased (Table 2). The patients having more than one metastasis and receiving RT displayed increase in Beck scores. This may be explained by the heightened awareness of patients for their disease after some doctor visits and RT sessions. For lung cancer when the difference between Beck- 0 and Beck1 exceeds 6 it is seen that risk of death increase (Table 3, Figure 1).

There is similar literature data on this issue. Researchers in the USA state that Worsening depression symptoms are associated with shorter survival rates for patients with lung cancer, especially those in the early stages of the disease (17). Jane Walker et al have found in their group of patients with breast, lung, colorectal, genitourinary, or gynaecological cancer that patients with lung cancer were more prone to major depression. Besides these patients may exhibit low adherence to treatment with lower benefits and shorter life span (18). Jue Chen et al have found the rate of depression to be $38 \%$ in 126 lung cancer patients and also these patients had lower quality of life, and displayed symptoms of fatigue.
Furthermore the same study showed that depression shortened survival rate by 90 days (19). According to previous studies, the 2-year survival rates of patients with bone metastasis were poor at $19-23 \%(20,21,22)$.

Our study implies that patients who are treated for cancer are more concerned about their future due to their depression. This leads to an increase in their anxiety and depression for the future $(23,24)$. The relatively small sample size may be one pitfall of our study. On the other hand depression in this study could be assessed for gender. This study is valuable for evaluating depression before and after RT in different cancer types.

Depression seen on the oncology patients is a psychological disease and can affect the primary disease's course negatively. Patients become more depressed when their treatment advances and that is documented by both Beck-0 and Beck-1 rising after initial RT in this study. During the course these patients seem to require psychiatric support. We hope our study helps develop new insights on psychiatric support for onchologic patients. It is important that safe and evidence-based palliative radiatiotherapy is considered.

\section{References}

1. Chambers A, Naumov G, Varghese H. Critical steps in hematogenous metastasis: an overview. Surg Oncol Clin N Am 2001; 10: 243255.

2. Coleman R. Metastatic bone disease: clinical features, pathophysiology and treatment strategies. Cancer Treat Rev 2001; 27: 165-176.

3. Selvaggi G, Scagliotti G. Management of bone metastases in cancer: a review. Clin Rev Oncol Hematol 2005; 56: 365-378.

4. Cecchini M, Wetterwald A, Pluijm G, Thalmann G. Molecular and biological mechanisms of bone metastasis: EAU Update Series 2005; 3: 214-226.

5. Janjan N. Radiation of bone metastases: conventional techniques and the role of systemic radiopharmaceuticals. Cancer 1997; 80: 16281645.

6. Firvida JL, Vinolas N, Munoz M. A critical factor in cancer management: A prospective comparative study of 400 patients. Age Ageing 1999; 28: 103-105.

7. Yancik R, Wesley MN, Ries LA. Comorbidity and age as predictors of risk for early mortality of male and female colon carcinoma patients: A population-based study. Cancer 1998; 82: 2123 2134.

8. Yancik R. Cancer burden in the aged: An epidemiologic and demographic overview. Cancer 1997; 80: 1273-1283. 
9. Alacacioglu A, Yavuzsen T, Dirioz M, Yilmaz U. Quality of life, anxiety and depression in Turkish breast cancer patients and in their husbands. Med Oncol 2009; 26(4): 415-419.

10. Chochinov H. Depression in cancer patients:Lancet Oncol 2001; 2: 499-505.

11. Paula JM, Sonobe HM, Nicolussi AC, Zago MM, Sawada NO. Symptoms of depression in patients with cancer of the head and neck undergoing radiotherapy treatment: a prospective study. [Article in English, Portuguese, Spanish] Rev Lat Am Enfermagem 2012; 20: 362-368.

12. Goreinstein C, Andrade L. Validation of Portuguese version of Beck Depression Inventory and State-trait Anxiety Inventory in Brazilian subjects:Braz J Med Biol Res 1996; 29: 453-457.

13. Sawada N.O, Juliana Maria de Paula Helena Megumi Sonobe, Marcia Maria Fontão Zago, Giselle Patricia Guerrero, Adriana Cristina Nicolussi. Depression, fatigue, and health-related quality of life in head and neck cancer patients: a prospective pilot study: Support Care Cancer. Published online 2012; 012: 1390-1392.

14. Hisli N. Beck Depresyon Envanteri'nin geçerliliği üzerine bir çalışma: Psikoloji Dergisi 1988; 6: 118122.

15. Sobin LH, Gospodarowicz MK, Wittekind C. TMN classification of malignant tumours: 7 th ed. London. Wiley Blackwell 2009.

16. Hung MS, Chen IC, Lee CP, et al. Incidence and risk factors of depression after diagnosis of lung cancer: A nationwide population-based study. Medicine (Baltimore) 2017; 96: e6864.

17. [No authors listed] Depression can shorten lung cancer survival rates. Nurs Stand 2016; 31: 17.
18. Walker J, Hansen CH, Martin P, et al. Prevalence, associations, and adequacy of treatment of major depression in patients with cancer: a crosssectional analysis of routinely collected clinical data. Lancet Psychiatry 2014; 1(5): 343-350.

19. Chen J, Li W, Cui L, et al. Chemotherapeutic Response and Prognosis among Lung Cancer Patients with and without Depression. J Cancer 2015; 6: 1121-1129.

20. Satin JR, Linden W, Phillips MJ. Depression as a predictor of disease progression and mortality in cancer patients: A metaanalysis. Cancer 2009; 115: 5349-5361.

21. Katagiri H, Takahashi M, Wakai K, et al. Prognostic factors and a scoring system for patients with skeletal metastasis: J Bone Jt Surg Br 2005; 87: 1505-1512.

22. Mizumoto M, Harada $\mathrm{H}$, Asakura $\mathrm{H}$, et al Prognostic factors and a scoring system for survival after radiation therapy for metastases to spinal column: a review of 544 patients at Shizuoka Cancer Center Hospital. Cancer 2008; 113: 2816-2822.

23. Jehn C. F. Influence of age, performance status, cancer activity, and IL-6 on anxiety and depression in patients with metastatic breast cancer: Breast Cancer Res Treat 2012; 136: 789794.

24. O'Connor M, White K, Kristjanson LJ, Cousins $\mathrm{K}$, Wilkes $\mathrm{L}$. The prevalence of anxiety and depression in palliative care patients with cancer in Western Australia and New South Wales. Med J Aust 2010; 193: 44-47. 